\title{
Impacto do tratamento hemodialítico em pacientes com doença renal crônica
}

\section{Impact of hemodialysis treatment in patients with chronic kidney disease}

\author{
Emanuela Pinheiro de Oliveira' • Giovana Mendes de Lacerda Leite ${ }^{2} \bullet$ Grayce Alencar Albuquerque $^{3}$ \\ Ana Paula Agostinho Alencar ${ }^{4} \bullet$ Rosa Maria Grangeiro Martins ${ }^{5} \bullet$ Izabel Cristina Santiago Lemos de Beltrão ${ }^{6}$ \\ Regina Petrola Bastos Rocha ${ }^{7}$ Dayanne Rakelly de Oliveira $^{8} \bullet$ Dailon de Araújo Alves 9
}

\begin{abstract}
RESUMO
Objetivo: compreender o impacto do tratamento hemodialítico na vida dos pacientes com Doença Renal Crônica. Métodos: estudo descritivo e exploratório de abordagem qualitativa, realizado com 15 pacientes de uma Unidade de Diálise e Transplante Renal, localizada na cidade de Crato-Ceará. Os dados coletados foram organizados através do Discurso do Sujeito Coletivo e analisados à luz da Teoria Adaptativa de Callista Roy. Resultados: os discursos dos participantes diante dos questionamentos possibilitaram identificar que a hemodiálise causa impacto direto na vida desses pacientes, interferindo no seu cotidiano, devido, principalmente às limitações sociais, que envolvem seu trabalho, hábitos alimentares e culturais, além do impacto na saúde mental. Conclusão: neste sentido, fomentar abordagens mais eficazes para o manejo terapêutico dos pacientes com doença renal crônica auxiliará a equipe de enfermagem na identificação dos problemas adaptativos mais frequentes, resultando em um cuidado mais resolutivo e congruente com as necessidades do paciente dialítico.

Palavras-chave: Doença Renal Crônica; Hemodiálise; Enfermagem.
\end{abstract}

\begin{abstract}
Objectives: to understand the impact of hemodialysis treatment on the life of patients with chronic kidney disease. Method: this is a descriptive and exploratory study with a qualitative approach, conducted with 15 patients from a Dialysis and Kidney Transplant Unit, located in the city of Crato-Ceará.The collected data were organized through Collective Subject Discourse and analyzed based on Callista Roy's Adaptive Theory. Results: the speeches of the participants in the face of the questions made it possible to identify that hemodialysis has a direct impact on the lives of these patients, interfering in their daily lives, mainly due to the social limitations that involve their work, eating and cultural habits, in addition to the impact on mental health. Conclusion: Promoting more effective approaches to the therapeutic management of patients with chronic kidney disease will assist the nursing staff in identifying the most frequent adaptive problems, resulting in more resolute care and congruent with the needs of the dialysis patient.
\end{abstract}

Keywords: Chronic Renal Disease; Hemodialysis; Nursing.

I Enfermeira. Membro da Associação Brasileira de Enfermagem (ABEN/CE). Integrante do Grupo de Pesquisa Tecnologias em Saúde no Sistema Único de Saúde (GPTSUS / CNPQ / URCA). Membro do Núcleo de Avaliação de Tecnologias em Saúde e Núcleo em Evidências (NATS / NEv). Residente em Enfermagem Obstétrica pela URCA.

2 Enfermeira. Mestre em Bioprospecção Molecular pela Universidade Regional do Cariri (URCA). Pós-graduanda em Saúde da Mulher pela Faculdade Futura. Membro do Grupo de Pesquisa Farmacognosia Quantitativa e Qualitativa pela URCA.

3 Enfermeira. Doutora em Ciências da Saúde pelo Programa de Pós-graduação da Faculdade de Medicina do ABC. Líder do Grupo de Pesquisa em Gênero, Sexualidade, Diversidade Sexual e Inclusão (GPESGDI). Coordenadora do Observatório de Violência e Direitos Humanos na Região do Cariri pela URCA. Docente do Quadro Efetivo da URCA.

4 Enfermeira. Mestre em Ciências da Saúde pela Faculdade de Medicina do ABC. Especialista em Saúde da Família e Prática Docente no Ensino Superior pela Faculdade Integrada de Patos (FIP). Coordenadora de Estágio do Curso Técnico em Vigilância em Saúde da Escola Técnica do SUS - ETSUS.

5 Enfermeira. Mestre em Saúde da Família pela Renasf / URCA. Especialista em Urgência e Emergência pela FIP. Especialista em Saúde Pública pela Universidade Estadual do Ceará (UECE). Especialista em Docência do Ensino Superior pela Universidade Federal do Ceará (UFC). Pós-graduanda em Estomaterapia pela URCA. Docente do Curso de Graduação em Enfermagem da Faculdade de Medicina Estácio - FMJ.

6 Enfermeira. Doutoranda pela Universidade Federal Rural de Pernambuco (UFRPE). Mestre em Bioprospecção Molecular pela URCA. Tutora e Docente do Programa de Residência Multiprofissional em Saúde Coletiva (PRMSC / URCA). Coordenadora do Projeto de Extensão PRÓSS-QUILOMBOLAS (PROEX / URCA). Docente Assistente do Curso de Graduação em Enfermagem da URCA.

7 Enfermeira. Doutoranda em Ciências da Saúde pela Faculdade de Medicina do ABC. Mestre em Desenvolvimento Regional Sustentável pela Universidade Federal do Ceará (UFC). Especialista em Gestão dos Sistemas e Serviços de Saúde pela Escola de Saúde Pública do Ceará (ESP/CE). Especialista em Saúde Pública pela Universidade Estadual do Ceará (UECE). Docente da Faculdade de Medicina Estácio de Juazeiro do Norte (FMJ).

8 Enfermeira. Doutora em Ciências Biológicas. Universidade Federal de Santa Maria (UFSM). Mestre em Bioprospecção Molecular pela URCA. PhD em Enfermagem pela Universidade Federal do Ceará (UFC). Professora Adjunta do Departamento de Enfermagem da URCA.

9 Enfermeiro. Mestre em Enfermagem pela Universidade Regional do Cariri (URCA). Especialista em Saúde da Família pela Faculdade Integrada de Patos (FIP). Pósgraduando em Estomaterapia pela URCA. Docente do Curso de Graduação em Enfermagem da Faculdade de Medicina Estácio (FMJ). E-mail: dailon.araujol2@ gmail.com. 


\section{INTRODUÇÃO}

A Doença Renal Crônica (DRC) é considerada atualmente um problema de saúde pública, com altas taxas de morbidade e mortalidade. Nos últimos anos, o número de pessoas com falência renal tem aumentado consideravelmente e, mesmo submetidas a tratamento adequado, o prognóstico ainda é insatisfatório e os custos são elevados. Anteriormente, à falência renal era tida como uma doença sem possibilidade de cura ou estabilização, hoje, sabe-se que existem algumas modalidades de tratamento que melhoram a qualidade de vida do paciente, dentre elas, a terapia hemodialítica ${ }^{(1)}$.

Segundo a Sociedade Brasileira de Nefrologia( ${ }^{(2)}$, a hemodiálise substitui a função dos rins em pacientes com DRC, mas não de forma completa, visto que o rim exerce outras funções além da filtragem sanguínea, como o controle da água corporal, da pressão arterial, e controle da saúde dos ossos através da produção de vitamina $D$.

$O$ cuidado de enfermagem aos pacientes em hemodiálise é ainda um grande desafio para o profissional enfermeiro, visto que é uma condição que acarreta limitações aos pacientes, fazendo com que eles dependam constantemente do cuidado desses profissionais, que devem estar aptos a lidarem com as diferentes reações expressas antes, durante e após as sessões, tornando-se necessário conhecer a realidade de cada um, para então traçar intervenções que os auxiliem nos cuidados diários ${ }^{(3)}$.

Nesta perspectiva, esse estudo foi mediado pelo seguinte questionamento: De que maneira a hemodiálise interfere na vida de pacientes com doença renal crônica? Além disso, objetivou-se compreender o impacto do tratamento hemodialítico em pacientes com DRC e as implicações para o cuidado da enfermagem.

\section{MÉTODOS}

Trata-se de um estudo descritivo-exploratório com abordagem qualitativa, realizado em uma Unidade de Diálise e Transplante Renal, localizada na cidade de Crato-Ceará, referência no atendimento e acompanhamento de pacientes com doença renal crônica. Por sua vez, o estudo ocorreu entre os meses de Julho à Outubro de 2017.

Os participantes da pesquisa foram pacientes diagnosticados com DRC de qualquer etiologia, que se encontravam no programa de hemodiálise na unidade de diálise já mencionada; e que atenderam aos seguintes critérios de inclusão: pacientes com idade igual ou superior a 18 anos, estar em tratamento hemodialítico há no mínimo seis meses e apresentar capacidade de compreensão preservada. $\mathrm{O}$ critério de exclusão adotado foi o fato do paciente apresentar alguma intercorrência clínica durante o processo de estabelecimento da pesquisa.

Posteriormente, os participantes que estiveram em consonância com os critérios de inclusão e que concor- daram com os objetivos da pesquisa, foram individualmente entrevistados por meio de um roteiro para entrevista semiestruturada, onde os diálogos provenientes das falas dos participantes foram gravados por meio de equipamento digital e transcritos.

Para organização dos dados foi utilizado o discurso do sujeito coletivo (DSC), o qual se trata de uma técnica de organização de dados qualitativos, onde é realizada a síntese de vários discursos, extraindo de discursos individuais suas ideias centrais (IC), expressões-chave $(\mathrm{ECH})$ e ou ancoragem (AC) de cada um, de acordo com a similaridade das ideias, e reunindo-as em um só discurso, que será redigido na primeira pessoa do singular e formará o discurso síntese ${ }^{(4)}$.

Para análise e interpretação dos dados foi utilizada a teoria de Callista Roy. Esta, em seu Modelo de Adaptação, propõe como objetivo da enfermagem a promoção da adequação das pessoas, individualmente ou no coletivo, em quatro modos de adaptação: modo adaptativo, físicofisiológico, identidade de autoconceito, interdependência e desempenho de papel, o que torna possível, a partir da implementação da teoria, colaborar para a saúde, a qualidade de vida e a morte com dignidade ${ }^{(5)}$.

Cabe ressaltar que o estudo respeitou as exigências éticas regulamentadoras de pesquisas envolvendo seres humanos (resolução $n^{\circ} 466 / / 2$ - CNS), sendo a pesquisa aprovada pelo Comitê de Ética e Pesquisa (CEP) da Universidade Regional do Cariri, sob o parecer de número 2.182.259.

\section{RESULTADOS E DISCUSSÃO}

\section{$O$ perfil da amostra: mapeando os participantes da pesquisa}

Participaram do estudo, I5 pacientes com diagnóstico de DRC, que realizavam hemodiálise na Unidade de Diálise e Transplante Renal selecionada como cenário da pesquisa, a pelo menos seis meses, dos quais 8 eram do sexo feminino e 7 do sexo masculino. A faixa etária variou entre 18 e 75 anos (Tabela I).

\section{Representação do discurso coletivo}

Durante a aplicação do roteiro de entrevistas, implementou-se 5 (cinco) perguntas para a posterior construção do DSC, valorizando o aspecto qualitativo do discurso e mensuração das ideias centrais. Salienta-se que foram reportados os DSC de maior representatividade no imaginário coletivo, seguindo a IC correspondente.

As perguntas utilizadas e as respectivas ideias centrais estão dispostas no quadro a seguir:

Quanto ao perfil da amostra e considerando que a maioria dos participantes da pesquisa apresentaram-se na faixa etária de 53 à 77 anos, vale ressaltar que alguns estudos revelaram que a idade avançada no início da tera- 
TABELA 1 - Distribuição das variáveis demográficas e clínicas, segundo cada grupo de tratamento Crato, CE, Brasil, 2017.

\begin{tabular}{|c|c|c|}
\hline Localidade & $\mathbf{N}$ & $\%$ \\
\hline Unidade de Diálise e Transplante Renal Dr. Raimundo Bezerra (UNIRIM), Crato - Ceará & 15 & 100 \\
\hline \multicolumn{3}{|l|}{ Sexo } \\
\hline Feminino & 8 & 53,36 \\
\hline Masculino & 7 & 46,64 \\
\hline \multicolumn{3}{|l|}{ Moradia } \\
\hline Zona Rural & 4 & 26,67 \\
\hline Zona Urbana & 11 & 77,33 \\
\hline \multicolumn{3}{|l|}{ Faixa Etária } \\
\hline $18-25$ & 2 & 13,3 \\
\hline $26-33$ & 2 & 13,3 \\
\hline $34-52$ & 3 & 20,00 \\
\hline $53-65$ & 4 & 26,7 \\
\hline $66-77$ & 4 & 26,7 \\
\hline \multicolumn{3}{|l|}{ Escolaridade } \\
\hline Ensino Fundamental Incompleto & 5 & 33,33 \\
\hline Ensino Fundamental Completo & 1 & 6,67 \\
\hline Ensino Médio Incompleto & 2 & 13,3 \\
\hline Ensino Médio Completo & 4 & 26,7 \\
\hline Sem Escolaridade & 3 & 20,00 \\
\hline \multicolumn{3}{|l|}{ Renda } \\
\hline 1 Salário mínimo & 14 & 93,33 \\
\hline 2 Salários mínimo & 1 & 6,67 \\
\hline \multicolumn{3}{|l|}{ Religião } \\
\hline Católico & 12 & 80,00 \\
\hline Evangélico & 2 & 13,3 \\
\hline Sem Religião & 1 & 6,7 \\
\hline \multicolumn{3}{|l|}{ Tempo de Tratamento na Unidade } \\
\hline $01-05$ anos & 10 & 66,66 \\
\hline $06-10$ anos & 2 & 13,34 \\
\hline $11-15$ anos & 3 & 20,00 \\
\hline
\end{tabular}

Fonte: Pesquisa direta na Unidade de Diálise. 2017.

QUADRO 1 - Perguntas (adaptadas) do roteiro de entrevista para representação das Ideias Centrais.

Perguntas

1. Você se considera uma pessoa com saúde?

2. Você sabe o que significa Doença Renal Crônica? E o que significa Hemodiálise?

3. Fale sobre as mudanças ocorridas em sua vida após o início do tratamento.

4. Você se sente capaz de prover o suporte pessoal, financeiro e que atenda às suas necessidades?

5. Quais são as suas expectativas em relação ao tratamento? E ao futuro? Comente.
Ideias Centrais

IC A: Sim, me considero saudável

IC B: Não, não me considero saudável

IC C: Sim, eu compreendo minha doença

IC D: Não, não compreendo minha doença

IC E: Sim, houve mudanças sociais e no estilo de vida

IC F: Não observei mudanças

IC G: Sim, sinto-me capaz de suprir o suporte financeiro necessário para minhas necessidades

IC H: Sim, sinto-me parcialmente capaz de suprir o suporte financeiro necessário para minhas necessidades

IC I: Não, eu me sinto incapaz de suprir o suporte financeiro necessário para minhas necessidades

IC J: Minhas expectativas são positivas com relação ao tratamento e ao futuro

IC K: Não tenho expectativas com relação ao tratamento e ao futuro

Fonte: Pesquisa direta na Unidade de Diálise, 2017.

pia, bem como outros fatores, estão diretamente relacionados à sobrevida dos pacientes, tendo um risco maior para cada ano a mais na idade que se inicia a hemodiálise, confirmando que a idade avançada, dentre outros fatores, está fortemente relacionada com a mortalidade ${ }^{(6)}$.

Relativo a análise do DSC, na pergunta I (Quadro I) é possível observar que duas ideias centrais (IC) foram obtidas a partir da análise dos discursos de cada participante. Alguns se consideraram com saúde (DSC A), e outros não se consideraram com saúde (DSC B). Isso pode estar relacionado à existência de muitos conceitos para saúde e doença: 
Eu me considero uma pessoa com saúde [...] tirando o problema renal, sou normal. [DSC A]

[...] saúde só se eu não tivesse nada renal, aí eu era sadia [...] os ploblemas nos rins atrapalha muita coisa. Eu não me considero com muita saúde, não! Eu sinto tanta coisa! Eu não posso mais trabalhar, não posso mais fazer nada. [DSC B]

Os conceitos sobre saúde e doença dependem do entendimento que se tem sobre si e de sua relação com o meio em que se está inserido; o que varia de acordo com a cultura de cada pessoa e lugar. A compreensão sobre a saúde e a doença; as crenças; valores e afetos são intrínsecos a cada ser humano, e representam o que eles sabem sobre si a partir das condições a que estão submetidos $^{(7)}$.

A pergunta 2, traz para os participantes da pesquisa a indagação sobre o significado, para eles, da DRC, bem como da hemodiálise. Neste cenário, foram obtidos duas IC (Quadro I), onde alguns relataram conhecimento sobre $o$ assunto em discussão, relacionando a doença aos rins e a hemodiálise à filtração do sangue; enquanto outros não demonstraram compreensão sobre a doença:

Doença renal é ser doente do rim [...] por que o rim para. Sente dor de cabeça, sente o corpo doído, pode ser causada por pressão alta, a minha começou por pressão alta. [A hemodiálise] é pra filtrar o sangue, né? hemodiálise é um jeito da gente ter um modo de vida melhor. A hemodiálise pra mim é tudo hoje, onde eu renasci [...] os rins não funciona mas ela ajuda. [DSC C]

Sei não. Eu não sei explicar, é muito complicada a doença renal. [Hemodiálise] eu acho bom, eu tive muito ruim, depois que eu comecei a fazer ela tá mais melhor, mas eu não sei pra que é que serve, não! [DSC D]

O conhecimento sobre doença, suas causas, sintomatologia, e a participação ativa nas decisões frente ao tratamento, juntamente com a equipe de profissionais são fundamentais para o equilíbrio emocional do paciente e para a qualidade da assistência prestada. $O$ sentimento de controle sobre o tratamento e a doença, solidifica a segurança e a confiança do paciente em si, tornando-o gestor da sua saúde ${ }^{(8)}$.

Referente à pergunta 3 , alusiva aos impactos que a hemodiálise causou na vida dos participantes, a maioria deles relatou não poder trabalhar. A atividade laboral de muitos, antes da doença, era diária e exigia muito esforço. Desta forma, a doença renal causou uma limitação ao trabalho, algumas vezes pela dinâmica do tratamento que leva os pacientes a se ausentarem frequentemente, ou pela atual condição física deles:

Não é que nem quando eu tinha minha saúde. Quando eu tinha minha saúde era mais diferente.
Mudou muita coisa. Você não pode viajar, você não pode trabalhar no pesado, né? [...] alimentação. Abri mão das coisas, parei de estudar. E liberdade também não tem. [DSC E]

Os pacientes com DRC perdem autonomia, no que diz respeito a experimentar novas restrições e limitações, passando a depender de um tratamento prolongado, doloroso, desgastante, porém, necessário. É importante ressaltar também, que a doença renal está relacionada com a incapacidade para o trabalho, resultando em dependência financeira, além do próprio tratamento de hemodiálise, o qual torna o indivíduo dependente ${ }^{(9)}$.

Os pacientes ainda relataram dificuldades em relação à necessidade de se deslocar para outra cidade para realizar as sessões de hemodiálise, a falta de transporte; 0 medo e o cansaço também foram citados. A rotina extenuante voltada para a realização das sessões, vários dias na semana, condiciona o paciente a um intenso desgaste.

Ainda nessa perspectiva, a literatura traz que as dificuldades de adesão ao tratamento dialítico, relatadas pelos pacientes, ocorrem principalmente pelas possíveis fontes de estresse que surgem durante 0 tratamento, como: dependência da Previdência Social, isolamento social, perda do emprego, impossibilidade de locomoção e passeios demorados, restrição parcial da atividade física, necessidade de adaptação à perda da autonomia, alterações da imagem corporal, além do medo de morrer. Tais condições tornam muitas vezes o paciente insatisfeito consigo mesmo, sentindo-se excluído ${ }^{(10)}$.

Além disso, destaca-se que um mesmo estímulo pode ocasionar comportamentos diferentes nos indivíduos, pelo fato de estar relacionado a fatores intrínsecos de enfrentamento, a teoria de adaptação de Roy torna possível reconhecer que as pessoas ao passarem por alguma patologia, mediante estímulos, podem desencadear respostas adaptativas ou não.

Para a presente pesquisa, alguns participantes mencionaram não observar mudanças significativas, contudo, tal reação pode representar, ainda, uma dificuldade adaptativa relacionada à negação. Cabe a equipe de saúde e ao enfermeiro contribuírem nesse processo, elaborando estratégias para o cuidado de enfermagem que englobem ações desde o momento de comunicação da necessidade de se realizar hemodiálise até o processo de reinserção social, através de orientações com objetivo de capacitar o paciente a criar mecanismos de enfrentamento visando diminuir as respostas não-adaptativas ${ }^{(I I)}$.

Todavia, é válido ressaltar que a doença passa a ter um sentido dentro da história de vida de cada pessoa. Em especial, o desenvolvimento de uma doença crônica que perdura por toda a vida e traz consigo algumas limitações físicas e sociais, além de mudanças significativas no cotidiano do paciente, principalmente no que diz 
respeito a atribuição de sentidos e significados atrelados a doença ${ }^{(12)}$. Assim, considerando que os indivíduos possuem inúmeras necessidades adaptativas, principalmente quando o padrão de saúde está alterado, o Modelo de Adaptação de Roy torna-se pertinente na prática do cuidado em Enfermagem baseado em conhecimentos específicos dessa área ${ }^{(13)}$.

Sobre a pergunta 4, frisa-se que os pacientes entrevistados não exerciam nenhuma atividade laboral, recebiam um salário mínimo proveniente da Seguridade Social, referente ao auxílio-doença. Parte dos pacientes relatou que o salário que recebiam supriam suas necessidades, contrário a estes, outros, afirmaram que um salário não dá para suprir todas as necessidades, visto que o gasto com medicação, pagamentos de água, luz, demandam boa parte do dinheiro recebido, variando de acordo com as despesas e demandas de cada um. No total, a maioria considerou que o salário recebido supre parcialmente ou não supre as suas necessidades.

Sim. Dá, só não dar para luxar [...] consegui o auxilio agora, aí melhorou mais, por que minha mãe que dava tudo. [DSC G]

Mais ou menos [...] Às vezes não dá, não! Mas a gente tem que abrir mão de muitas coisas! Não dá porque é muita coisa, água, luz. [DSC H]

Não dá, com certeza não, não dá! As coisa tudo é cara, né? Não dá, e o cabra compra muito remédio. Dá não! [DSC I]

Por sua vez, o tratamento dialítico condiciona o paciente a uma rotina restrita, esse fato interfere na capacidade de manter vínculos empregatícios formais, também pelas complicações e limitações físicas advindas com o problema renal. Essa situação provoca uma diminuição da autoestima dos indivíduos que se tornam dependentes financeiramente e a responsabilidade de manter a família é delegada a outra pessoa ${ }^{(9)}$.

O modo de adaptação e desempenho de papel, segundo Roy, se refere aos papéis os quais cada pessoa atua na sociedade, no sentido de preservar a integridade social, ou seja, saber quem se é em relação aos outros. No geral, as pessoas desempenham papéis primários, secundários ou terciários, estes levados a cabo por comportamentos instrumentais, sentimentos e atitudes de uma pessoa frente ao desempenho de um papel ${ }^{(5)}$.

Acerca da pergunta 5 , referente às expectativas com o tratamento, destaca-se que parte dos pacientes que inicia a hemodiálise manifesta a esperança no retorno do funcionamento dos rins, mesmo com a constatação de que não há cura para a DRC, ou mesmo na perspectiva do transplante renal:

Eu espero conseguir o transplante, porque bom [total] não fica mais não, né? [Mas] eu tenho fé, muita fé em Deus que um dia meu rim vai voltar ao me- nos um ao normal, eu tenho muita fé. Eu [até] já transplantei, transplantei! Fiquei um ano e quatro meses, aí rejeitou devido minha imunidade. Bom, eu espero tá sempre bem, melhorar, né? Deus é quem sabe. [DSC J]

Nesta perspectiva, é válido destacar o aspecto da fél religião, que emerge no discurso dos participantes. A religião oferece suporte emocional e social, uma espécie de religação, e motivação para cuidar da saúde, adotando estilos de vida mais saudáveis. Portanto, os profissionais de saúde precisam conhecer as crenças que tornam o paciente e seus familiares dispostos a aceitar o tratamento(14). Além disso, a esperança é uma estratégia de enfrentamento, uma perspectiva positiva em relação ao futuro. Ela incentiva $\circ$ indivíduo para o bem-estar espiritual, estando relacionada à sobrevida e a força para o enfrentamento de problemas. A experiência de adoecer, quando enfrentada com esperança, faz o indivíduo depositar energia na expectativa de melhora da sua saúde e do bem-estar ${ }^{(15)}$.

Assim, o modelo teórico de Roy considera o cuidado de enfermagem como uma interação constante com o meio, focalizando na necessidade de transformar os problemas de adaptação dos pacientes em respostas positivas. Tornando-se, portanto, relevante a aplicação desse modelo ao cuidado de pacientes com doenças crônicas em tratamento de diálise de forma a contribuir para promoção da adaptação e integridade da pessoa ${ }^{(16)}$.

Nesse contexto, o enfermeiro, através do processo de enfermagem de Roy, pode contribuir para a promoção da adaptação positiva do cliente. Os sentimentos vivenciados por pessoas com uma doença crônica são diversos, dentre eles a regressão, a perda da autoestima, a insegurança, a ansiedade, a negação da situação presente e a depressão ${ }^{(9)}$.A partir da forma de viver, da situação de adoecimento, intrínseca a cada paciente, e dos mecanismos de defesa e adaptativos usados por ele, teremos ou não uma resposta ativa do doente e da família no controle apropriado da doença ${ }^{(18)}$.

Desta forma, a investigação da história do paciente; a observação do seu comportamento; questionamentos sobre a sua forma de ser e pensar antes e posterior à doença e ao tratamento; seus desejos; expectativas em relação ao futuro e conhecimento do diagnóstico são fundamentais para poder ajudá-lo.

Diante de tantos conflitos, é necessário que o paciente se sinta otimista, resiliente e à vontade para expressar suas ansiedades e medos, estabelecendo vínculo terapêutico não só com a família, mas com os profissionais envolvidos no seu cuidado. Quanto mais emponderado e integrado com sua própria vida, melhor este paciente enfrentará sua doença ${ }^{(8)}$. 


\section{CONCLUSÃO}

A pesquisa revelou que a diálise afeta o cotidiano e a vida desses pacientes, causando também alterações significativas tanto nos aspectos psicossociais como físicos, visto que, diante da realidade da DRC e da necessidade de um tratamento complexo, são geradas desordens emocionais resultantes do processo de adaptação à nova realidade, principalmente em relação às limitações sociais, envolvendo seu trabalho, seus hábitos alimentares e seu convívio familiar.

Também, foi possível observar que surgem diversos sentimentos e frustrações que necessitam de auxílio de profissionais da saúde, capacitados, que reconheçam as particularidades de cada paciente e como cada um vivencia este processo de adaptação. Fenômeno esse, que vai ao encontro do que preconiza a teoria de Roy, onde os processos adaptativos precisam ser valorizados no contexto do cuidar, facilitando por sua vez, uma assistência dita integral e resolutiva.

Por fim, espera-se que esse trabalho possa contribuir para a realização de estudos posteriores que valorizem, de fato, o que é denominado de assistência holística. 


\section{REFERÊNCIAS}

I. Martins LCN, Magnabosco P, Pereira MER. Mulheres em tratamento hemodialítico: qualidade de vida e assistência de enfermagem. Rev. Aten. Saúde. [Internet]. 2019 [acesso em I3 out 2020]; v. 17, n. 59, p. 62-72. DOI: 10.13037/ras. voll $7 \mathrm{n} 59.5790$.

2. Sociedade Brasileira de Nefrologia. Hemodiálise. Editor do Portal: Dr.Alexandre Silvestre Cabral. Copyright. [Internet]. 2017 [acesso em 2l jun 2018] Disponível em: https://sbn. org.br/publico/tratatamentos/hemodialise/.

3. Silva FRC, Santos MS, Pereira RG, Sousa PV. Nursing and complications frequently during hemodialysis: literature review. Reon Facema [Internet]. 2016 [acesso em I 3 out 2020]; JulSet; 2(3): 238-24I. Disponível em: https://www.facema.edu. br/ojs/index.php/ReOnFacema/article/viewFile/84/5I.

4. Figueiredo MZA, Chiari BM, Goulart BNG. Discurso do Sujeito Coletivo: uma breve introdução à ferramenta de pesquisa qualiquantitativa. Distúrb Comum [Internet]. 2013 [acesso em 22 jun 2018]; 25(I): I29-36. Disponível em: https://revistas.pucsp.br/dic/article/view/ /493 I/I I I 39.

5. Coelho SMS, Mendes IMDM. Da pesquisa a prática de enfermagem aplicando o modelo de adaptação de Roy. Esc Anna Nery (impr.) [Internet]. 20II [acesso em 22 jun 20I8]; 15(4): 845-850. Disponível em: https://www.scielo.br/pdf/ ean/vI5n4/a26vI5n4.pdf.

6. Teixeira FIR, Lopes MLH, Silva GAS, Santos RF. Sobrevida dePacientes em Hemodiálise em um Hospital Universitário. J. Bras. Nefrol. [Internet] 2014 [acesso em 22 jun 2018]; 37(I): 64-7I. DOI: 10.5935/0I0I-2800.201500I0.

7. Nascimento FAF. Uma contribuição às reflexões sobre os aspectos emocionais e o papel do psicólogo na Hemodiálise. Rev. SBPH [Internet]. 2013 [acesso em 23 jun 2018]; I6(I): 70-87. Disponível em: http://pepsic.bvsalud.org/pdf/rsbph/ vI6nI/vI6nla05.pdf.

8. Silva RAR, Souza VL, Oliveira GJN, Silva BCO, Rocha CCT, Holanda JRR. Estratégias de enfrentamento utilizadas por pacientes renais crônicos em tratamento hemodialítico. Esc Anna Nery [Internet]. 2016 [acesso em I 4 out 2020]; 20(I):|47-I54. DOI: I0.5935/|4|48145.20160020 .

9. Evangelista TR, Lisboa AR, Dantas AEF, Oliveira IB, Anjos EU. Repercussão do tratamento hemodialítico na vida dos pacientes com insuficiência renal crônica no Sertão Paraibano. Revista Brasileira de Educação e Saúde [Internet]. 2016 [acesso em 16 out 2020]; v.6, n.4, p.01-09, out-dez. Disponí- vel em: https://editoraverde.org/gvaa.com.br/revista/index. php/REBES/article/view/4595/397I.

10. Machado GRG, Pinhati GR.Tratamento de diálise em pacientes com insuficiência renal crônica. Cadernos UniFOA [Internet]. 2014 [acesso em 20 jun 2018]; n. 26: I37-48. Disponível em: http://web.unifoa.edu.br/cadernos/edicao/26/I37-I48.pdf.

I I. Monteiro AKC, Costa CPV, Campos MOB, Monteiro AKC. Aplicabilidade da teoria de Callista Roy no cuidado de enfermagem ao estomizado. Rev Enferm Atenção Saúde [Internet]. 2016 [acesso em 20 jun 20 I8]; 5(I):84-92. Disponível em: http://seer.uftm.edu.br/revistaeletronica/index.php/ enfer/article/view/l625/pdf.

12. Pichinelli JJ, Milagres CS. Percepção da insuficiência renal crônica e enfrentamento de pacientes jovens em tratamento hemodialítico. Enfermagem Brasil [Internet]. 2018 [acesso em I4 out 2020]; I7(3):I82-9. Disponível em: https:// portalatlanticaeditora.com.br/index.php/enfermagembrasil/article/view/II 77.

13. Medeiros LP, Souza MBC, Sena JF, Melo MDM, Costa JWS, Costa IKF. Modelo de Adaptação de Roy: revisão integrativa dos estudos realizados à luz da teoria. Rev Rene [Internet]. 2015 [acesso em 2 abr 20I8]; I6(I): I32-40. DOI: I0.15253/2|75-6783.20150001000|7.

I4. Souza LS, Branco PCC, Branco ABAC. Percepções de psicólogas frente às crenças religiosas de pessoas hospitalizadas: estudo fenomenológico. Rev. Nufen: Phenom. Interd. [Internet]. 2019 [acesso em 16 out 2020]; I I(I), 7I-85, jan. - abr. DOI: 10.26823/RevistadoNUFEN.voll I .n0 I artigo46.

15. Ottaviani AC,Souza EM,Drago NC,Mendiondo MSZ,Pavarini SI, Orlandi FS. Esperança e espiritualidade de pacientes renais crônicos em hemodiálise: estudo correlacional. Rev. Latino-Am. Enfermagem [Internet]. 2014 [acesso em $2 \mathrm{abr}$ 20 I8]; 22(2): 248-54. DOI: I 0. I 590/0 I04-I I 69.3323.2409.

16. Frazão CMFQ, Fernandes MICD, Nunes MGM, Sá JD, Lopes MVO, Lira ALBC. Componentes do modelo teórico de Roy em pacientes submetidos à hemodiálise. Rev. Gaúcha Enferm [Internet]. 2013 [acesso em 2 abr 20 I8]; 34(4): 45-52. Disponível em: https://www.scielo.br/pdf/rgenf/v34n4/06.pdf.

17. Rudnicki T. Doença renal crônica: vivência do paciente em tratamento de hemodiálise. Contextos Clínicos [Internet]. 2014 [acesso em 3 abr 20I8]; 7(I): I05- I6. DOI: 10.40I3/ ctc.2014.7I.10.

Recebido: 2020-09-15

Aceito: 2020-10-27 